Research Article

\title{
Outcome Assessment According to the Thickness and Direction of the Acellular Dermal Matrix after Implant-Based Breast Reconstruction
}

\author{
Joon Hur and Hyun Ho Han \\ Department of Plastic Surgery, Asan Medical Center, University of Ulsan College of Medicine, Seoul, Republic of Korea \\ Correspondence should be addressed to Hyun Ho Han; tripleh1952@gmail.com
}

Received 20 May 2021; Revised 11 September 2021; Accepted 1 November 2021; Published 16 November 2021

Academic Editor: Michele Maruccia

Copyright (c) 2021 Joon Hur and Hyun Ho Han. This is an open access article distributed under the Creative Commons Attribution License, which permits unrestricted use, distribution, and reproduction in any medium, provided the original work is properly cited.

\begin{abstract}
Purpose. The acellular dermal matrix plays an important role in reinforcing thin mastectomy skin and repositioning the implant in prosthetic breast reconstruction. As the concept of prepectoral plane has become widespread, the role of the acellular dermal matrix has become increasingly important. However, evidences and standards for appropriate thickness and direction during placement remain insufficient. This study is aimed at testing the assumption that differences in the acellular dermal matrix thickness and orientation during placement may affect surgical outcomes including the incidence of postoperative complications. Methods. This was a retrospective single-centered analysis of 43 patients (50 breasts) who underwent implantbased reconstruction with MegaDerm ${ }^{\circledR}$ (L\&C Bio, Seoul, Korea) and 23 patients (23 breasts) who underwent implant-based reconstruction with DermACELL ${ }^{\circledR}$ (LifeNet Health, Virginia Beach, VA, USA), two types of human-derived acellular dermal matrix. All surgeries were performed by a single surgeon. Demographic variables, surgery-related factors, and complications were compared between a thick matrix group $(1.5-2.3 \mathrm{~mm})$ and a thin matrix group $(1.0-1.5 \mathrm{~mm})$. The same processes were performed in the nonreverse and reverse matrix insertion groups. Results. Baseline demographics and surgery-related data were summarized according to matrix thickness and direction. There were no significant intergroup differences in the demographic variables such as history of smoking, radiation, or chemotherapy. The mean drain volume was significantly higher in the thick matrix group than that in the thin matrix group $(p=0.0445)$. However, there were no significant differences in overall complication rates by matrix thickness $(p=0.3139)$. Additionally, there were no significant differences in complications between the nonreverse and reverse matrix insertion groups $(p=0.538)$. Conclusion. Our findings suggest that patients with a thick acellular dermal matrix need a prolonged period for engraftment. However, the thickness did not directly affect the surgical outcomes between the thick and thin matrix groups. Likewise, the orientation in which the acellular dermal matrix was inserted did not affect the surgical outcomes including postoperative complications.
\end{abstract}

\section{Introduction}

Acellular dermal matrix $(\mathrm{ADM})$ is a tissue graft processed from cadaver, animal, or synthetic materials. ADM is commonly used in prosthetic breast reconstruction for its pliability, strength, tissue integration, and potential role in the mitigation of capsular contracture [1]. Specifically, the $\mathrm{ADM}$ acts as a scaffold for autologous cell growth and revascularization, providing an extra layer of soft-tissue support for the prosthesis [2].
The use of ADM in breast reconstruction is gradually expanding $[3,4]$. Using ADM, the thickness of the mastectomy flap can be reinforced, the position of the implant is stabilized, and complications such as capsular contracture can be reduced [5-10]. As the use of ADM increases and the concept of prepectoral breast reconstruction becomes widely accepted in prosthetic reconstruction [11, 12], more drawbacks of ADM, such as seroma and infection of the engraftment issue, have been reported [13-20]. Also, since several types of $\mathrm{ADM}$ are available from porcine, bovine, 

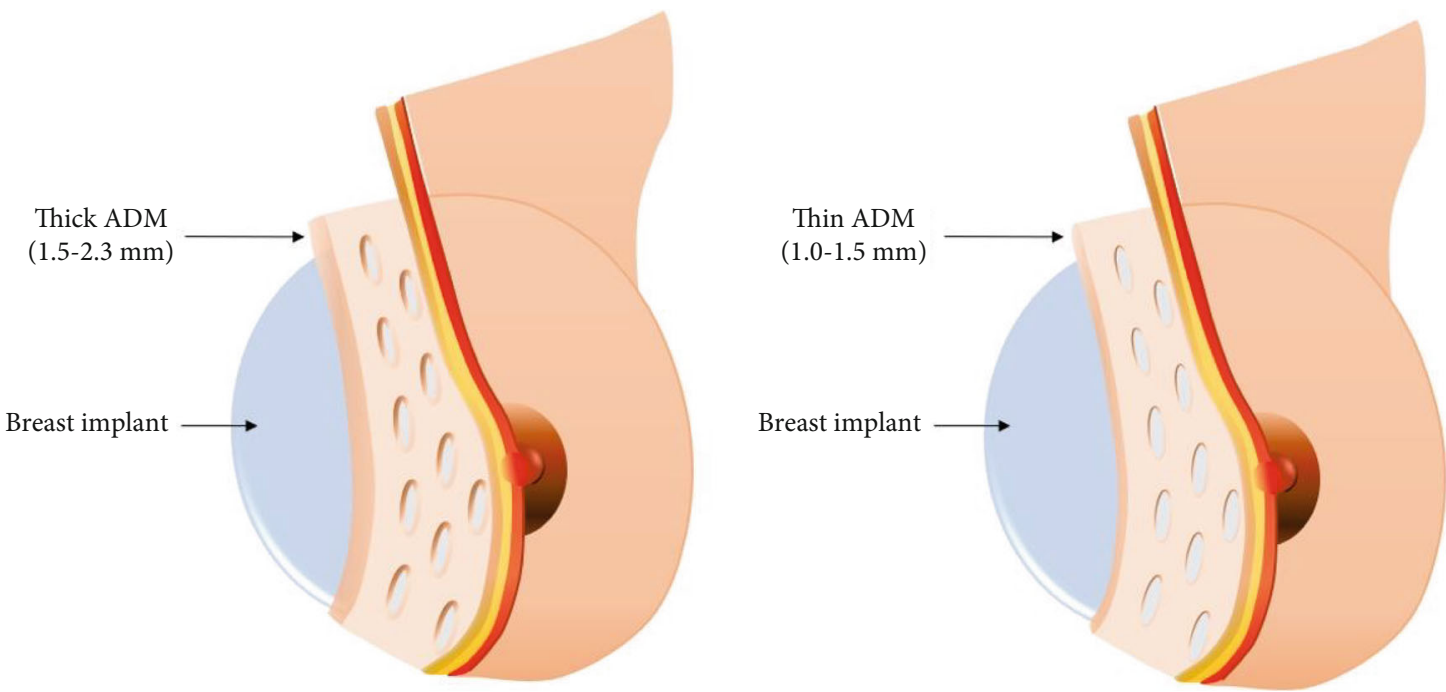

FiguRE 1: Different acellular dermal matrix thicknesses.

and human sources from different manufacturers [21], there are a variety of physical and biochemical characteristics [22], and doctors may use an ADM according to either the manufacture protocols or their own protocols based on surgical environment and experience. Therefore, it is necessary to gather data regarding the different techniques and their outcomes to standardize protocols for ADM use.

This study is aimed at describing how ADM thickness and insertion direction of two types of human-derived ADM affect implant-based breast reconstruction outcomes. We hypothesized that a thicker ADM would prolong biointegration and result in poorer outcomes in breast reconstruction. We also hypothesized that there would be differences in outcomes based on ADM insertion direction since the ADM has different anterior and posterior sides (dermal and basement, respectively).

\section{Patients and Methods}

2.1. Population and Study Design. A retrospective chart review was performed to identify patients who had undergone direct-to-implant breast reconstruction after mastectomy using ADM between April 2017 and March 2020 in a single center. All the surgeries were performed by a single surgeon.

MegaDerm ${ }^{\circledR}$ (L\&C Bio, South Korea) and DermACELL ${ }^{\circledR}$ (Stryker, USA) ADM were used in this study. The group of patients treated with MegaDerm $^{\circledR}$ was divided into two subgroups by ADM thickness. The thin ADM was 1.0$1.5 \mathrm{~mm}$ thick, while the thick ADM was $1.5-2.3 \mathrm{~mm}$ thick (Figure 1). The group of patients treated with DermACELL ${ }^{\circledR}$ was divided into two subgroups by direction of insertion with the basement membrane side contacting the implant in one group and the dermal side contacting the implant in the other group (Figure 2). Preoperatively, the types of ADM used in each operation were selected randomly regardless of the thickness of the mastectomy flap or the state of flap circulation. Patients who underwent simultaneous additional or secondary procedures during the operation were excluded.
Patients who underwent an implant change or expander-toimplant were also excluded from the study.

2.2. Data Collection. Baseline data included age, body mass index, smoking history, obesity, history of neoadjuvant radiotherapy, history of adjuvant radiotherapy, history of neoadjuvant chemotherapy, and history of adjuvant chemotherapy.

Surgery-related factors were also collected and consisted of mastectomy specimen weight, inserted implant size, ADM area, time to suction drain removal, total drainage volume, mastectomy method, implant insertion plane, implant texture, and breast cancer laterality. In the case of prepectoral implant insertions, ADM coverage was performed only at the anterior aspect of implants.

The following postoperative complications were also assessed for at least 6 months: capsular contracture, rippling, nipple sloughing, mastectomy flap necrosis, seroma, hematoma, red breast syndrome, implant rotation, and animation deformity. We defined major complications as those requiring surgical interventions and minor complications as those that did not.

2.3. Statistical Analysis. Mean, standard deviation, median value, and range were calculated for all continuous variables, while absolute frequencies and percentages were calculated for all categorical variables. All categorical variables were calculated and compared using the chi-squared test or Fisher's exact test, while all continuous variables were calculated and compared using the Mann-Whitney $U$ test or Student's $t$-test. To compare the complication rates between groups, Fisher's exact test and generalized estimating equation model for logit link were applied. Odds ratios and 95\% confidence intervals were also calculated. The statistical analyses were conducted using IBM SPSS (IBM Corp., Armonk, NY, USA). The criterion for significance was $\alpha<$ 0.05 (one-sided). The criterion for negating the preliminary differences between groups was $\alpha<0.05$. 

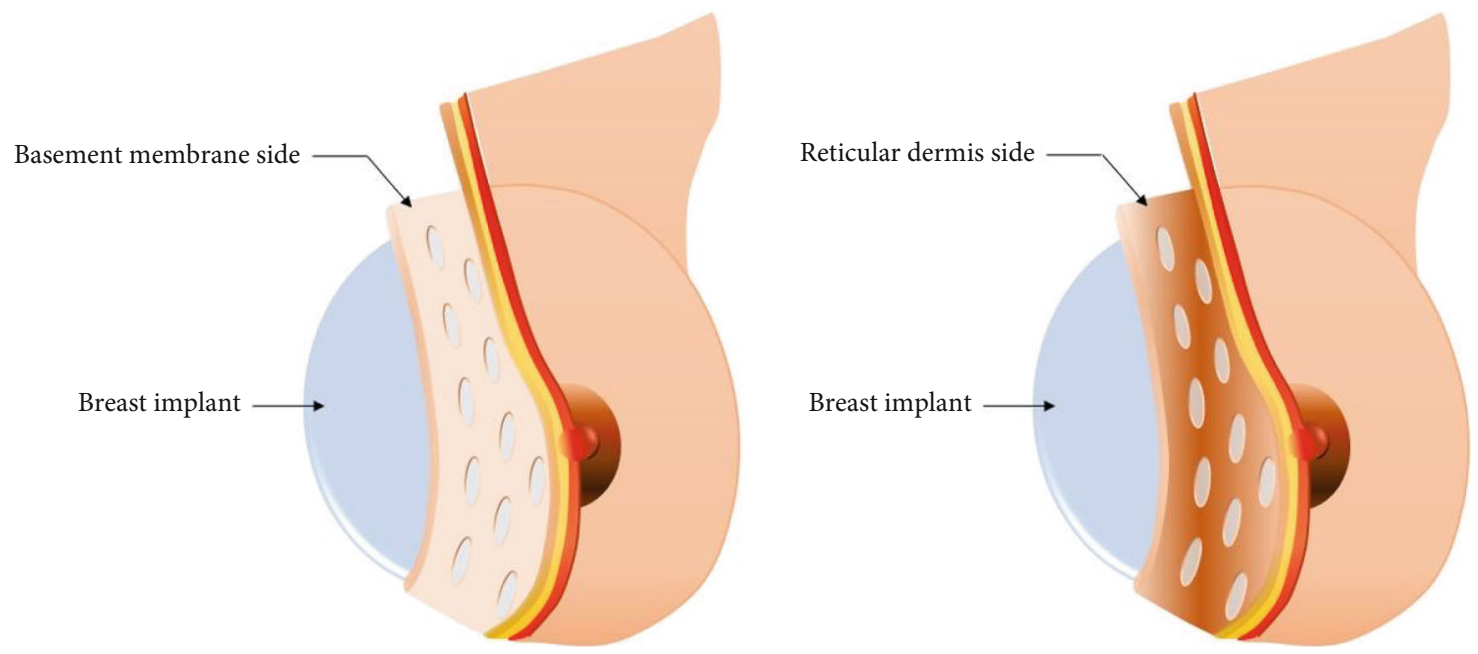

FIGURE 2: Different acellular dermal matrix insertion directions.

\section{Results}

3.1. Results by ADM Thickness. Patient demographics according to ADM thickness are summarized in Table 1.

There were no significant differences between the patients in the thick and thin ADM groups regarding mean age, body mass index, smoking history, obesity, history of chemotherapy, or history of radiotherapy (Table 1). There were no significant differences between the thick and thin $\mathrm{ADM}$ groups in surgery-related factors including time to drain removal, operation site, mastectomy type, implant insertion plane, implant texture, or axillary lymph node dissection (Table 2). However, there was a significantly higher mean drain volume in the thick ADM group $(994.73 \mathrm{~mL})$ than that in the thin ADM group $(723.35 \mathrm{~mL} ; p=0.0445)$.

The complications of the two groups are described in Table 3. There were no significant differences in all types of complications between the thick and thin ADM groups. Although the $p$ value of the mastectomy flap necrosis rate did not indicate significance $(p=0.06123)$, the incidence of mastectomy flap necrosis tended to be higher in the thick ADM group (23\%) than in the thin ADM group (4\%).

3.2. Results by ADM Orientation. Demographics by ADM orientation are summarized in Table 4 . There were no significant differences between the nonreverse and reverse ADM insertion groups in mean age, body mass index, smoking history, obesity, history of chemotherapy, or history of radiotherapy (Table 4). The mean drain volume in the nonreverse ADM insertion group was $580.24 \mathrm{~mL}$, while that of the reverse ADM insertion group was $524.67 \mathrm{~mL}(p>0.999)$. In addition, there were no significant differences between the nonreverse and reverse ADM insertion groups regarding surgery-related factors, including time to drain removal, operation site, mastectomy type, implant insertion plane, implant texture, and axillary lymph node dissection (Table 5).

In the nonreverse ADM insertion group, the minor complication rate was $7.14 \%(1 / 14)$ without major complications. In the reverse $\mathrm{ADM}$ insertion group, the minor complication rate was $22.22 \%(2 / 9)$, with no major compli-
TABLE 1: Patient baseline demographics and concurrent treatments.

\begin{tabular}{lccc}
\hline & Thick ADM & Thin ADM & $p$ value \\
\hline No. of patients & 23 & 20 & \\
Age $(\mathrm{yr})$ & & & 0.3538 \\
$\quad$ Mean \pm SD & $45.65 \pm 10.52$ & $42.80 \pm 9.24$ & \\
$\quad$ Range & $25-61$ & $24-61$ & \\
BMI $\left(\mathrm{kg} / \mathrm{m}^{2}\right)$ & & & 0.112 \\
$\quad$ Mean \pm SD & $24.48 \pm 7.01$ & $21.90 \pm 2.60$ & \\
$\quad$ Range & $17.69-50.54$ & $17.58-27.01$ & \\
Smoking $(n, \%)$ & $1(4.35 \%)$ & $3(15 \%)$ & 0.3235 \\
Obesity $(n, \%)$ & $2(8.7 \%)$ & $0(0 \%)$ & 0.4906 \\
Radiotherapy $(n, \%)$ & $6(26.09 \%)$ & $5(25 \%)$ & 0.9351 \\
Preoperative & 0 & 0 & \\
Postoperative & $6(26.09 \%)$ & $5(25 \%)$ & 0.9351 \\
Chemotherapy $(n, \%)$ & $10(43.48 \%)$ & $12(60.0 \%)$ & 0.2797 \\
Preoperative & $5(21.74 \%)$ & $1(5 \%)$ & 0.1918 \\
Postoperative & $10(43.48 \%)$ & $11(55 \%)$ & 0.4509 \\
Pre+post & $5(21.71 \%)$ & $0(0 \%)$ & 0.0511 \\
\hline
\end{tabular}

ADM: acellular dermal matrix; SD: standard deviation; BMI: body mass index. Obesity was defined as $\mathrm{BMI}>25 \mathrm{~kg} / \mathrm{m}^{2}$.

cations. There were no statistically significant differences between the nonreverse and reverse ADM insertion groups in terms of complications (Table 6).

\section{Discussion}

This study is aimed at describing how thickness and insertion direction of two types of human-derived ADM affected implant-based breast reconstruction outcomes. Our results indicate that there are no significant differences in intraoperative outcomes according to thickness or insertion direction.

Since ADM was first introduced in 2001, it has become increasingly common in prosthetic breast reconstruction. Specifically, the prepectoral technique has played an important role in the accelerated ADM use in the past 3-4 years [23-25], and ADM accounts for a greater portion of 
TABLE 2: Surgery-related factors.

\begin{tabular}{|c|c|c|c|}
\hline & Thick ADM & Thin ADM & $p$ value \\
\hline No. of breasts & 26 & 24 & \\
\hline Implant size (cc) & & & 0.176 \\
\hline Mean \pm SD & $272.462 \pm 100.224$ & $234.375 \pm 95.432$ & \\
\hline Range & $160-480$ & $95-450$ & \\
\hline Mastectomy weight (g) & & & 0.5999 \\
\hline Mean \pm SD & $365.208 \pm 273.339$ & $295.958 \pm 161.124$ & \\
\hline Range & $125-1150$ & $96-828$ & \\
\hline $\operatorname{ADM}$ size $\left(\mathrm{m}^{2}\right)$ & & & 0.067 \\
\hline Mean \pm SD & $121.615 \pm 33.555$ & $109.750 \pm 34.132$ & \\
\hline Range & $70-192$ & 75-192 & \\
\hline Jackson-Pratt drain (mL) & & & 0.0445 \\
\hline Mean \pm SD & $994.731 \pm 539.652$ & $723.354 \pm 365.827$ & \\
\hline Range & $268-2737$ & $320.5-1588$ & \\
\hline Time to drain removal (day) & & & 0.5297 \\
\hline Mean \pm SD & $19.385 \pm 6.350$ & $18.375 \pm 4.735$ & \\
\hline Range & $9-31$ & $12-27$ & \\
\hline Operation site $(n, \%)$ & & & 0.5547 \\
\hline Left & $13(50 \%)$ & $10(41.67 \%)$ & \\
\hline Right & $13(50 \%)$ & $14(58.33 \%)$ & \\
\hline Mastectomy type $(n, \%)$ & & & 0.4585 \\
\hline NSM & $17(65.38 \%)$ & $18(75 \%)$ & \\
\hline Radial incision & $10(58.82 \%)$ & $11(61.11 \%)$ & \\
\hline Periareolar incision & $4(23.53 \%)$ & $4(22.22 \%)$ & \\
\hline Lateral incision & $3(17.65 \%)$ & $3(16.67 \%)$ & \\
\hline SSM & $9(34.62 \%)$ & $6(25 \%)$ & \\
\hline $\operatorname{ALND}(n, \%)$ & $21(80.77 \%)$ & $19(79.17 \%)$ & $>0.999$ \\
\hline Insertion plane $(n, \%)$ & & & 0.9819 \\
\hline Prepectoral & $12(46.15 \%)$ & $11(45.83 \%)$ & \\
\hline Subpectoral & $14(53.85)$ & $13(54.17 \%)$ & \\
\hline Implant texture $(n, \%)$ & & & 0.5791 \\
\hline Smooth & $10(38.46 \%)$ & $13(54.16 \%)$ & \\
\hline Microtexture & $15(57.69 \%)$ & $10(41.67 \%)$ & \\
\hline Macrotexture & $1(3.85 \%)$ & $1(4.17 \%)$ & \\
\hline
\end{tabular}

ADM: acellular dermal matrix; SD: standard deviation; ALND: axillary lymph node; SSM: skin sparing mastectomy; NSM: nipple areolar skin sparing mastectomy.

surgeries compared with synthetic mesh in recent prosthetic breast reconstructions. However, a synthetic mesh remains an alternative tool to cover breast prosthetics. Some studies suggest that the use of a synthetic mesh improves aesthetic results and reduces the incidence of capsular contracture, very similar to the role of ADM but with lower cost [26]. In addition, the use of a synthetic mesh has some advantages such as reducing surgical time for implant positioning, thereby lowering exposure time and risk of infection [27]. Therefore, the current research is aimed at elucidating the effectiveness of ADM, describing the associated complications, and exploring the reasons for the increased cost [28].

Thus, whether ADM thickness affects prosthetic breast reconstruction outcomes requires consideration. Generally, a thick ADM provides robust mechanical support, but there are also some concerns with engraftment. Prolonged engraftment may increase the risk of complications like seroma or infection and may induce differentiated skin textures [13-20]. In other words, a thin ADM is more easily incorporated and less likely to cause the complications such as seroma or infection. Determining whether ADM thickness affects prosthetic breast reconstruction outcomes can provide surgeons with evidence when deciding whether to use an ADM in their surgeries.

This study revealed no significant differences in complication rates between patients with thick and thin ADM. In terms of demographic and surgery-related factors, only the mean drain volume differed between the groups. By 
TABLE 3: Postoperative complications.

\begin{tabular}{|c|c|c|c|c|c|}
\hline & Thick ADM & Thin ADM & $\mathrm{OR}^{*}$ & $95 \% \mathrm{CI}$ & $p$ value \\
\hline Total complication $(n, \%)$ & & & 0.5352 & $\begin{array}{c}0.1585- \\
1.8069\end{array}$ & 0.3139 \\
\hline No complication & $13(50 \%)$ & $16(66.67 \%)$ & & & \\
\hline Minor* & $9(34.62 \%)$ & $8(33.33 \%)$ & & & \\
\hline Major* & $4(15.38 \%)$ & $0(0 \%)$ & & & \\
\hline Capsular contracture & $1(3.85 \%)$ & $0(0 \%)$ & $\mathrm{NE}$ & & \\
\hline Rippling & $1(3.85 \%)$ & $3(12.5 \%)$ & 3.6379 & $\begin{array}{l}0.3450- \\
38.3556\end{array}$ & 0.2826 \\
\hline Nipple sloughing & $3(11.54 \%)$ & $2(8.33 \%)$ & 0.3509 & $\begin{array}{c}0.0335- \\
3.6744\end{array}$ & 0.3821 \\
\hline Rotation & $1(3.85 \%)$ & $1(4.17 \%)$ & 1.2589 & & 0.8711 \\
\hline Animation & $0(0 \%)$ & $2(8.33 \%)$ & $\mathrm{NE}$ & & \\
\hline RBS & $1(3.85 \%)$ & $1(4.17 \%)$ & 1.0935 & $\begin{array}{l}0.0636- \\
18.8027\end{array}$ & 0.9509 \\
\hline Mastectomy flap necrosis & & & 0.1288 & $\begin{array}{c}0.0146- \\
1.1371\end{array}$ & 0.0651 \\
\hline No complication & $20(76.92 \%)$ & $23(95.83 \%)$ & & & \\
\hline Minor & $2(7.69 \%)$ & $1(4.17 \%)$ & & & \\
\hline Major & $4(15.38 \%)$ & $0(0 \%)$ & & & \\
\hline Seroma & & & 0.5259 & $\begin{array}{c}0.0438- \\
6.3136\end{array}$ & 0.6123 \\
\hline No complication & $24(92.31 \%)$ & $23(95.83 \%)$ & & & \\
\hline Minor & $2(7.69 \%)$ & $1(4.17 \%)$ & & & \\
\hline Major & $0(0 \%)$ & $0(0 \%)$ & & & \\
\hline Hematoma & & & $\mathrm{NE}$ & & \\
\hline No complication & $26(100 \%)$ & $24(100 \%)$ & & & \\
\hline Minor & $0(0 \%)$ & $0(0 \%)$ & & & \\
\hline Major & $0(0 \%)$ & $0(0 \%)$ & & & \\
\hline
\end{tabular}

${ }^{*}$ Major: the complications which needed secondary surgical procedures. ${ }^{*}$ Minor: the complications which did not need secondary surgical procedures. ADM: acellular dermal matrix; OR: odds ratio; CI: confidence interval; NE: not estimated; RBS: red breast syndrome.

TABle 4: Patient baseline demographics and concurrent treatments.

\begin{tabular}{|c|c|c|c|}
\hline & Reverse (-) & Reverse (+) & $p$ value \\
\hline No. of patients & 14 & 9 & \\
\hline Age (yr) & & & 0.336 \\
\hline Mean \pm SD & $45.88 \pm 7.48$ & $49.33 \pm 8.11$ & \\
\hline Range & $36-62$ & $32-59$ & \\
\hline BMI $\left(\mathrm{kg} / \mathrm{m}^{2}\right)$ & & & 0.557 \\
\hline Mean \pm SD & $22.2 \pm 3.2$ & $21.58 \pm 3.11$ & \\
\hline Range & $15.88-26.56$ & $16.08-28.37$ & \\
\hline Smoking $(n, \%)$ & 0 & 0 & \\
\hline Obesity $(n, \%)$ & 0 & 0 & \\
\hline Radiotherapy $(n, \%)$ & $3(21.43 \%)$ & 0 & 0.253 \\
\hline Preoperative & 0 & 0 & \\
\hline Postoperative & $3(21.43 \%)$ & 0 & 0.235 \\
\hline Chemotherapy $(n, \%)$ & $6(42.86 \%)$ & $1(11.11 \%)$ & 0.176 \\
\hline Preoperative & $2(14.29 \%)$ & 0 & 0.502 \\
\hline Postoperative & $5(35.71 \%)$ & $1(11.11 \%)$ & 0.34 \\
\hline Pre+post & $1(7.14 \%)$ & 0 & $>0.999$ \\
\hline
\end{tabular}

ADM: acellular dermal matrix; SD: standard deviation; BMI: body mass index. Obesity was defined as BMI $>25 \mathrm{~kg} / \mathrm{m}^{2}$. 
TABLE 5: Surgery-related variables.

\begin{tabular}{|c|c|c|c|}
\hline & Reverse (-) & Reverse $(+)$ & $p$ value \\
\hline No. of breasts & 14 & 9 & \\
\hline Implant size (cc) & & & 0.282 \\
\hline Mean \pm SD & $254.642 \pm 83.526$ & $216.667 \pm 61.644$ & \\
\hline Range & $125-400$ & $130-300$ & \\
\hline Mastectomy weight (g) & & & 0.361 \\
\hline Mean \pm SD & $283.429 \pm 149.942$ & $225.667 \pm 81.974$ & \\
\hline Range & $55-547$ & $90-342$ & \\
\hline $\operatorname{ADM}$ size $\left(\mathrm{m}^{2}\right)$ & & & 0.625 \\
\hline Mean \pm SD & $121.143 \pm 20.698$ & $117.333 \pm 41.569$ & \\
\hline Range & $80-160$ & $64-192$ & \\
\hline Jackson-Pratt drain (mL) & & & $>0.999$ \\
\hline Mean \pm SD & $580.243 \pm 342.052$ & $524.667 \pm 99.919$ & \\
\hline Range & $126-1588.4$ & $336-637$ & \\
\hline Time to drain removal (day) & & & 0.734 \\
\hline Mean \pm SD & $15.214 \pm 3.051$ & $15.556 \pm 3.712$ & \\
\hline Range & $12-22$ & $11-20$ & \\
\hline Operation site $(n, \%)$ & & & 0.68 \\
\hline Left & $8(57.14 \%)$ & $4(44.44 \%)$ & \\
\hline Right & $6(42.86 \%)$ & $5(55.56 \%)$ & \\
\hline Mastectomy type $(n, \%)$ & & & $>0.999$ \\
\hline NSM & $7(50.0 \%)$ & $5(55.56 \%)$ & \\
\hline SSM & $7(50.0 \%)$ & $4(44.44 \%)$ & \\
\hline $\operatorname{ALND}(n, \%)$ & $3(21.43 \%)$ & $2(22.22 \%)$ & $>0.999$ \\
\hline Insertion plane $(n, \%)$ & & & 0.343 \\
\hline Prepectoral & $12(85.71 \%)$ & $6(66.67 \%)$ & \\
\hline Subpectoral & $2(14.29 \%)$ & $3(33.33 \%)$ & \\
\hline Implant texture $(n, \%)$ & & & 0.232 \\
\hline Smooth & $13(92.86 \%)$ & $8(88.89 \%)$ & \\
\hline Microtexture & $1(7.14 \%)$ & $1(11.11 \%)$ & \\
\hline Macrotexture & 0 & 0 & \\
\hline
\end{tabular}

SD: standard deviation; ADM: acellular dermal matrix; ALND: axillary lymph node dissection; SSM: skin sparing mastectomy; NSM: nipple areolar skin sparing mastectomy.

confirming the increased Jackson-Pratt (JP) drain volume in the thick ADM group, we concluded that a thicker ADM needs a longer drain time. However, despite the increased drain volume and time to engraftment, the prosthetic breast reconstruction outcomes were not affected.

At the outset of our study, we expected that incision types in nipple sparing mastectomy would be a factor affecting outcomes. A total of three types of incisions were included in the present study, specifically, radial, periareolar, and lateral incisions. Fortunately, there was no difference in the proportion of complications by the incision type. Even though there were cases of nipple slough, no additional surgical procedures were needed. However, we could not generalize the influence of incision type due to the small numbers of cases included.

The two sides of ADM have different biologic characteristics [29]. The surface side serves as the basement membrane, and the opposite side contains the reticular dermis
(Figure 3). Manufacturers usually instruct surgeons to place the reticular dermis side on the site to be engrafted, which means the basement membrane side faces the implant, while the reticular dermis side faces the mastectomy skin. However, it is possible to place the ADM inside out due to a surgeon's lack of experience or mistakes during surgery. Therefore, it is important to elucidate the effects of ADM insertion orientation on outcomes. If ADM insertion orientation does not affect outcomes and the role of the basement membrane is not significant, manufacturers can produce multiple sheets of ADM from a thicker dermis area such as the back or head. Therefore, determining whether the basement membrane is necessary to maintain the shape of the breast by helping to maintain the dermal strength later is important for clinical practice.

In the present study, we found no significant differences in baseline demographics, surgery-related factors, or complication rates between the nonreverse and reverse ADM 
TABLe 6: Postoperative complications.

\begin{tabular}{|c|c|c|c|c|c|}
\hline & Reverse (-) & Reverse (+) & $\mathrm{OR}^{*}$ & $95 \% \mathrm{CI}$ & $p$ value \\
\hline Total complication $(n, \%)$ & $1(7.14 \%)$ & $2(22.22 \%)$ & 0.269 & $0.021-3.519$ & 0.538 \\
\hline Minor* & 1 & 2 & & & \\
\hline Major* & 0 & 0 & & & \\
\hline Capsular contracture & 0 & 0 & $\mathrm{NE}$ & & \\
\hline Rippling & 0 & $2(22.22 \%)$ & NE & & \\
\hline Nipple sloughing & 0 & 0 & NE & & \\
\hline Rotation & 0 & 0 & NE & & \\
\hline Animation & 0 & 0 & $\mathrm{NE}$ & & \\
\hline RBS & $1(7.14 \%)$ & 0 & $\mathrm{NE}$ & & \\
\hline Mastectomy flap necrosis & 0 & 0 & $\mathrm{NE}$ & & \\
\hline Seroma & 0 & 0 & $\mathrm{NE}$ & & \\
\hline Hematoma & 0 & 0 & $\mathrm{NE}$ & & \\
\hline
\end{tabular}

* Major: the complications which needed secondary surgical procedures. ${ }^{*}$ Minor: the complications which did not need secondary surgical procedures. ADM: acellular dermal matrix; OR: odds ratio; CI: confidence interval; NE: not estimated; RBS: red breast syndrome.

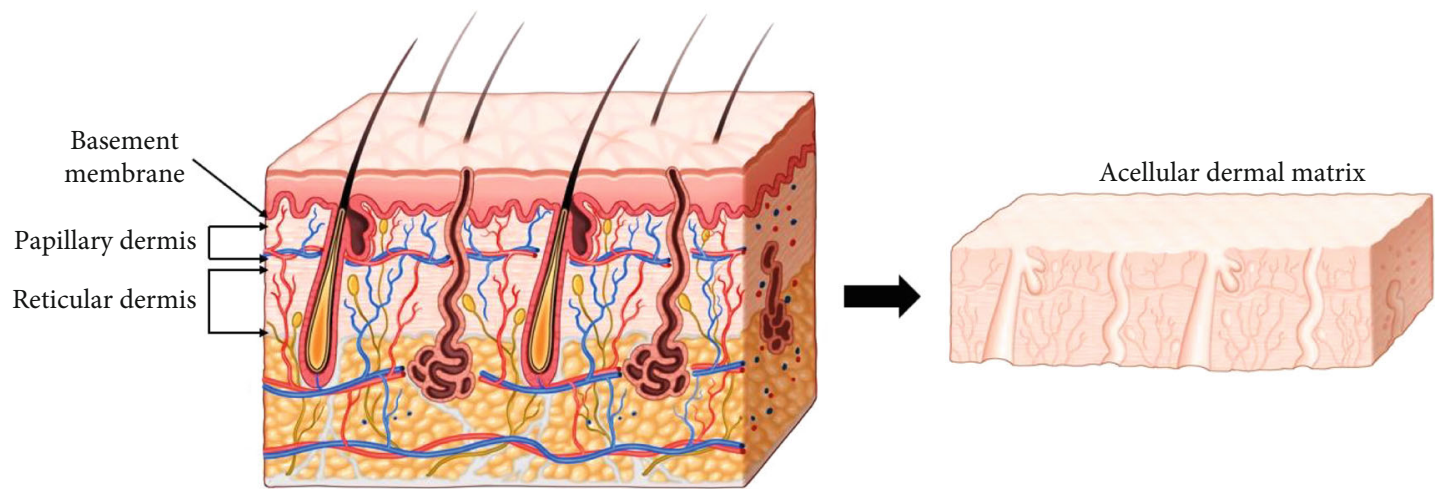

Figure 3: The surface side is the basement membrane, and the opposite side is the reticular dermis. After the decellularization process, only the structure of the dermis is utilized.

insertion groups. Thus, we found no relation between ADM thickness and orientation with prosthetic breast reconstruction outcomes. It is important to note that ADM thickness and orientation did not affect engraftment. These results suggest that ADM can be used with more flexibility. For example, a thicker ADM can be applied to provide stronger mechanical support in cases of patients with very thin skin tissue, while a thinner ADM can be applied to obtain more pleasing aesthetic results in patients with a thick mastectomy flap or those undergoing a risk-reducing mastectomy.

Our study has several limitations. First, our study used a retrospective design, and a small number of patients were included. In addition, there may be a possible selection bias since all the patients were of the same race; since Asian patients tend to be slim, there was a lower possibility of including patients with large breasts. Thus, the possibility of complications may be underestimated.

\section{Conclusion}

Our results suggest that a thick ADM requires a prolonged engraftment period due to the large drainage volume. This, however, did not directly affect the surgical outcomes between patients receiving a thick versus thin ADM. Likewise, the orientation in which the ADM was inserted did not affect surgical outcomes or the incidence of complications.

\section{Data Availability}

The data used to support the findings of this study are restricted by the Asan Medical Center Institutional Review Board in order to protect patient privacy. Data are available from HH Han, tripleh1952@gmail.com, for researchers who meet the criteria for access to confidential data.

\section{Conflicts of Interest}

The authors declare that they have no conflicts of interest.

\section{References}

[1] M. Zenn, M. Venturi, T. Pittman et al., "Optimizing outcomes of postmastectomy breast reconstruction with acellular dermal matrix: a review of recent clinical data," Eplasty, vol. 17, article e18, 2017. 
[2] N. Sobti, E. Ji, R. L. Brown et al., "Evaluation of acellular dermal matrix efficacy in prosthesis-based breast reconstruction," Plastic and Reconstructive Surgery, vol. 141, no. 3, pp. 541-549, 2018.

[3] C. R. Albornoz, P. B. Bach, B. J. Mehrara et al., "A paradigm shift in U.S. breast Reconstruction," Plastic and Reconstructive Surgery, vol. 131, no. 1, pp. 15-23, 2013.

[4] A. S. Colwell, B. Damjanovic, B. Zahedi, L. Medford-Davis, C. Hertl, and W. G. Austen Jr., "Retrospective review of 331 consecutive immediate single-stage implant reconstructions with acellular dermal matrix: indications, complications, trends, and costs," Plastic and Reconstructive Surgery, vol. 128, no. 6, pp. 1170-1178, 2011.

[5] J. Y. Yang, C. W. Kim, J. W. Lee, S. K. Kim, S. A. Lee, and E. Hwang, "Considerations for patient selection: prepectoral versus subpectoral implant-based breast reconstruction," Archives of Plastic Surgery, vol. 46, no. 6, pp. 550-557, 2019.

[6] M. Maruccia, R. Elia, A. Gurrado et al., "Skin-reducing mastectomy and pre-pectoral breast reconstruction in large ptotic breasts," Aesthetic Plastic Surgery, vol. 44, no. 3, pp. 664-672, 2020.

[7] S. L. Spear, M. Seruya, M. W. Clemens, S. Teitelbaum, and M. Y. Nahabedian, "Acellular dermal matrix for the treatment and prevention of implant-associated breast deformities," Plastic Surgical Nursing, vol. 37, no. 2, pp. 76-87, 2017.

[8] M. Schmitz, M. Bertram, U. Kneser, A. K. Keller, and R. E. Horch, "Experimental total wrapping of breast implants with acellular dermal matrix: a preventive tool against capsular contracture in breast surgery?," Journal of Plastic, Reconstructive \& Aesthetic Surgery, vol. 66, no. 10, pp. 1382-1389, 2013.

[9] A. Cheng, C. Lakhiani, and M. Saint-Cyr, "Treatment of capsular contracture using complete implant coverage by acellular dermal matrix: a novel technique," Plastic and Reconstructive Surgery, vol. 132, no. 3, pp. 519-529, 2013.

[10] E. M. Kobraei, R. Cauley, M. Gadd, W. G. Austen Jr., and E. C. Liao, "Avoiding breast animation deformity with pectoralissparing subcutaneous direct-to-implant breast reconstruction," Plastic and Reconstructive Surgery. Global Open, vol. 4, no. 5, article e708, 2016.

[11] A. R. Carmichael and K. Mokbel, "Evolving trends in breast surgery: oncoplastic to onco-aesthetic surgery," Archives of Plastic Surgery, vol. 43, no. 2, pp. 222-223, 2016.

[12] U. Wazir and K. Mokbel, "The evolving role of pre-pectoral ADM-assisted implant-based immediate breast reconstruction following skin-sparing mastectomy," American Journal of Surgery, vol. 216, no. 3, pp. 639-640, 2018.

[13] T. JoAnna Nguyen, J. N. Carey, and A. K. Wong, "Use of human acellular dermal matrix in implant- based breast reconstruction: evaluating the evidence," Journal of Plastic, Reconstructive \& Aesthetic Surgery, vol. 64, no. 12, pp. 15531561, 2011.

[14] R. H. Schnarrs, C. M. Carman, C. Tobin, S. A. Chase, and K. A. Rossmeier, "Complication rates with human acellular dermal matrices: retrospective review of 211 consecutive breast reconstructions," Plastic and Reconstructive Surgery. Global Open, vol. 4, no. 11, article e1118, 2016.

[15] H. Sbitany, "Important considerations for performing prepectoral breast reconstruction," Plastic and Reconstructive Surgery, vol. 140, no. 6S, pp. 7S-13S, 2017.

[16] A. Singla, A. Singla, E. Lai, and D. Caminer, "Subcutaneously placed breast implants after a skin-sparing mastectomy: do we always need ADM?," Plastic and Reconstructive Surgery. Global Open, vol. 5, no. 7, article e1371, 2017.

[17] S. Y. Kim and S. I. Bang, "Impact of acellular dermal matrix (ADM) use under mastectomy flap necrosis on perioperative outcomes of prosthetic breast reconstruction," Aesthetic Plastic Surgery, vol. 41, no. 2, pp. 275-281, 2017.

[18] G. Ho, T. J. Nguyen, A. Shahabi, B. H. Hwang, L. S. Chan, and A. K. Wong, "A systematic review and meta-analysis of complications associated with acellular dermal matrix-assisted breast reconstruction," Annals of Plastic Surgery, vol. 68, no. 4, pp. 346-356, 2012.

[19] J. Y. S. Kim, A. A. Davila, S. Persing et al., "A meta-analysis of human acellular dermis and submuscular tissue expander breast reconstruction," Plastic and Reconstructive Surgery, vol. 129, no. 1, pp. 28-41, 2012.

[20] M. L. Mangialardi, M. Salgarello, P. Cacciatore, I. Baldelli, and E. Raposio, "Complication rate of prepectoral implant-based breast reconstruction using human acellular dermal matrices," Plastic and Reconstructive Surgery. Global Open, vol. 8, no. 12, article e3235, 2020.

[21] M. Specht, S. Kelm, and U. Mirastschijski, "Eignung biologischer azellulärer dermaler matrices als Hautersatz," Handchirurgie, Mikrochirurgie, Plastische Chirurgie, vol. 52, no. 6, pp. 533-544, 2020, (in German).

[22] M. G. Onesti, M. Maruccia, G. di Taranto et al., "Clinical, histological, and ultrasound follow-up of breast reconstruction with one-stage muscle-sparing "wrap" technique: a singlecenter experience," Journal of Plastic, Reconstructive \& Aesthetic Surgery, vol. 70, no. 11, pp. 1527-1536, 2017.

[23] M. L. Mangialardi, M. Salgarello, I. Baldelli, and E. Raposio, "Prepectoral implant pocket conversion in breast reconstruction," JPRAS Open, vol. 26, pp. 12-25, 2020.

[24] M. Chandarana and S. Harries, "Multicentre study of prepectoral breast reconstruction using acellular dermal matrix," BJS Open, vol. 4, no. 1, pp. 71-77, 2020.

[25] R. Reitsamer, F. Peintinger, F. Klaassen-Federspiel, and A. Sir, "Prepectoral direct-to-implant breast reconstruction with complete ADM or synthetic mesh coverage - 36-months follow-up in 200 reconstructed breasts," Breast, vol. 48, pp. 32-37, 2019.

[26] D. Casella, J. Kaciulyte, F. Lo Torto et al., "'To pre or not to pre": introduction of a prepectoral breast reconstruction assessment score to help surgeons solving the decisionmaking dilemma. Retrospective results of a multicenter experience," Plastic and Reconstructive Surgery, vol. 147, no. 6, pp. 1278-1286, 2021.

[27] F. Lo Torto, M. Marcasciano, J. Kaciulyte et al., "Prepectoral breast reconstruction with TiLoop ${ }^{\circledR}$ Bra Pocket: a single center prospective study," European Review for Medical and Pharmacological Sciences, vol. 24, no. 3, pp. 991-999, 2020.

[28] M. Y. Nahabedian, "Acellular dermal matrix, the breast, and the FDA," Plastic and Reconstructive Surgery, vol. 145, no. 2, pp. 463e-464e, 2020.

[29] J. L. Owens, K. G. Cordell, M. Amornporncharoen, and A. A. Palaiologou, "Histologic evaluation of acellular dermal matrix allografts in humans," Clinical Advances in Periodontics, vol. 7, no. 3, pp. 122-127, 2017. 